\title{
The divergence of Van Hove's model and its consequences
}

\author{
Fulvio Sbisà* \\ Departamento de Física Teórica, Universidade do Estado do Rio de Janeiro, \\ CEP 20550-013, Rio de Janeiro - RJ - Brazil
}

\begin{abstract}
We study a regularized version of Van Hove's 1952 model, in which a quantum field interacts linearly with sources of finite width lying at fixed positions. We show that the central result of Van Hove's 1952 paper on the foundations of Quantum Field Theory, the orthogonality between the spaces of state vectors which correspond to different values of the parameters of the theory, disappears when a well-defined model is considered. We comment on the implications of our results for the contemporary relevance of Van Hove's article.
\end{abstract}

Keywords: Quantum Physics; Quantum Field Theory; Unitarily Inequivalent Representations; Non-separable Hilbert Spaces.

\section{Introduction}

The existence of Unitarily Inequivalent Representations (UIR) of the Canonical Commutation Relations (CCR) is an unavoidable feature of quantum systems with an infinite number of degrees of freedom, since in that case the Stone-von Neumann theorem $[1,2]$ does not hold. Although their existence were known at least before World War II [3] (to von Neumann, at any rate), the community of physicists started to become more widely aware of their relevance mainly due to the works of Van Hove [4] and Friedrichs $[5,6]$. In particular, Van Hove proposed a simple model where the existence of UIR was revealed by the circumstance that the spaces of state vectors relative to different values of the model's parameters are orthogonal. This showed how (borrowing Wightman and Schweber's words [7]) "other representations of the commutation rules are not pathological phenomena whose

*fulviosbisa@gmail.com ; https://orcid.org/0000-0002-6341-1785 . 
construction requires mathematical trickery, since they occur in the most elementary examples of field theory". See $[8,9]$ for a historical recollection and for a discussion of the connection of Van Hove's model with Haag's theorem $[10,11]$.

The model considered by Van Hove is apparently innocent, consisting of several point-like charges lying at fixed positions which interact with a neutral and relativistic massive scalar field. The whole analysis rests on the remarkable fact that the quantum equations of motions can be solved exactly, without resorting to the perturbative approach. While acknowledging that the model is unrealistic, Van Hove conjectured that the spaces of state vectors relative to different values of the coupling constant would remain orthogonal, or at least different, also in a more realistic QFT. From a mathematical point of view, however, the model is singular, since the exact solutions are divergent. Indeed the analysis becomes possible only after neglecting an infinite contribution to the ground state energy of the system (besides the already discarded ground state energy of the quantum harmonic oscillators), operation which is in no way justified in the article.

In light of its historical relevance, our aim in this paper is to perform a critical scrutiny of the article's analysis. Two points in particular are examined: the first is whether the phenomenon of orthogonality of the spaces of state vectors would still be present if we considered a less unrealistic modelization of the sources, such as one where the sources have finite but non-zero radius or a charge profile rapidly decaying at infinity. The second, related, point is whether it is possible to modify the model in such a way to regularize the above mentioned divergence while preserving the non-trivial phenomenology. A strong correlation between the divergence and the orthogonality phenomenon, in fact, would raise the suspicion that the latter is just an artifact of an ill-based model. Conversely, the absence of such a correlation would provide an a posteriori justification for the procedure of neglecting the infinite energy contribution. An important part of this investigation is to understand the physical meaning of the divergence.

The paper is structured as follows. In section 2 we consider a regularized version of Van Hove's model and investigate the physical meaning of the above mentioned divergence. The orthogonality of the spaces of state vectors in the regularized model is investigated in section 3, and the implications of our results are discussed in section 4 . We gather our conclusions in section 5 .

\section{The model and its generalization}

Although in the original model [4] the scalar field interacts with an arbitrary (finite) number of sources, we focus here on the special case of two sources, which we believe to be sufficiently representative of the general situation. 
We refer to the recent exposition [12] of Van Hove's article for a detailed reference.

To recall its main features, the model consists of two point-like sources placed respectively at the positions $\mathbf{y}_{1}$ and $\mathbf{y}_{2}$ (with $\mathbf{y}_{1} \neq \mathbf{y}_{2}$ strictly), interacting with a real, relativistic scalar field $\phi(t, \mathbf{x})$ of mass $m$ via an interaction term linear in $\phi$ and proportional to the charge of the source. The system is enclosed in a cubic box of large volume with periodic boundary conditions, so the field belongs to the space of (square-integrable) functions periodic on a three-dimensional cubic lattice. We indicate with $L$ the length of the edges of the primitive cell $\mathscr{P}$, and $V=L^{3}$ indicates its volume, so the reciprocal lattice, which we indicate with $\mathcal{R}$, is again a cubic lattice whose primitive cell has edges of length $2 \pi / L$ and volume equal to $(2 \pi)^{3} / V$.

\subsection{Spatially distributed sources}

As anticipated above, the model is idealized in various ways. For one thing, the sources create the field but don't react to it, their positions being fixed ("infinite mass" approximation). Besides, they have no internal structure and have zero spatial width, being point-like. The second property seems the most delicate, in light of the fact that strictly speaking it is not consistent to define a quantum field sharply at a point [13].

We therefore consider a slightly less idealized model, in which the sources have a finite spatial width and an internal structure. This can be done easily by introducing two smooth (real) functions $\rho_{1}$ and $\rho_{2}$ of compact support, normalized to one and centered around the origin. The idea is that the product $q_{i} \rho_{i}$, suitably shifted in space, plays the role of charge density distribution of the $i$-th source. We assume that the diameter of the supports of $\rho_{1}$ and $\rho_{2}$ is much smaller than the length $\left\|\mathbf{y}_{1}-\mathbf{y}_{2}\right\|$, and that their supports remain contained in the interior of $\mathscr{P}$ when translated by the vectors $\mathbf{y}_{1}$ and $\mathbf{y}_{2}$. We then consider the refined model given by

$$
\begin{aligned}
H & =H_{0}+g H_{\mathrm{i}} \\
H_{0} & =\frac{1}{2} \int_{\mathscr{P}}\left(\pi^{2}+|\vec{\nabla} \phi|^{2}+m^{2} \phi^{2}\right) d^{3} x \\
H_{\mathrm{i}} & =\sum_{i=1}^{2} q_{i} \int_{\mathscr{P}} \rho_{i}\left(\mathbf{x}-\mathbf{y}_{i}\right) \phi(t, \mathbf{x}) d^{3} x
\end{aligned}
$$

where the coupling constant $g$ is adimensional and natural units $\hbar=c=1$ are used. The charge density distributions are fixed, which means that both the position of the sources and their internal structure still do not react to the field. The description is still quite idealized, but slightly less unrealistic. 
Naively speaking, Van Hove's model is reproduced when the functions $\rho_{1}$ and $\rho_{2}$ "tend to a Dirac delta".

\subsection{Quantization}

The quantization proceeds exactly as in [4]. We keep the derivations short and refer to [12] for a more detailed exposition. Upon canonically quantizing the model (2.1)-(2.3) in the Schrödinger picture, the field operator and its canonically conjugate momentum read

$$
\begin{aligned}
& \hat{\phi}(\mathbf{x})=\frac{1}{\sqrt{2 V}} \sum_{\mathbf{k} \in \mathcal{R}} \frac{1}{\sqrt{\omega_{k}}}\left(\hat{a}_{\mathbf{k}} e^{i \mathbf{k} \cdot \mathbf{x}}+\hat{a}_{\mathbf{k}}^{\dagger} e^{-i \mathbf{k} \cdot \mathbf{x}}\right) \\
& \hat{\pi}(\mathbf{x})=\frac{-i}{\sqrt{2 V}} \sum_{\mathbf{k} \in \mathcal{R}} \sqrt{\omega_{k}}\left(\hat{a}_{\mathbf{k}} e^{i \mathbf{k} \cdot \mathbf{x}}-\hat{a}_{\mathbf{k}}^{\dagger} e^{-i \mathbf{k} \cdot \mathbf{x}}\right),
\end{aligned}
$$

where $\omega_{k}=\sqrt{m^{2}+k^{2}}$ and $k=\|\mathbf{k}\|$. The sum over $\mathcal{R}$ is in practice a sum over $\mathbb{Z}^{3}$, since the wave vectors are of the form $\mathbf{k}=\mathbf{n} 2 \pi / L$ with $\mathbf{n} \in \mathbb{Z}^{3}$. Imposing the field and momentum operators to satisfy the "continuum" CCR

$$
[\hat{\phi}(\mathbf{x}), \hat{\phi}(\mathbf{y})]=[\hat{\pi}(\mathbf{x}), \hat{\pi}(\mathbf{y})]=0 \quad, \quad[\hat{\phi}(\mathbf{x}), \hat{\pi}(\mathbf{y})]=i \delta(\mathbf{x}-\mathbf{y})
$$

amounts to postulating the following commutation relations for the operators $\hat{a}$ and $\hat{a}^{\dagger}$

$$
\left[\hat{a}_{\mathbf{k}}, \hat{a}_{\mathbf{k}^{\prime}}\right]=\left[\hat{a}_{\mathbf{k}}^{\dagger}, \hat{a}_{\mathbf{k}^{\prime}}^{\dagger}\right]=0, \quad\left[\hat{a}_{\mathbf{k}}, \hat{a}_{\mathbf{k}^{\prime}}^{\dagger}\right]=\delta_{\mathbf{k}, \mathbf{k}^{\prime}} .
$$

Neglecting in $\hat{H}_{0}$ the (infinite) ground state energy of the oscillators we obtain

$$
\begin{aligned}
& \hat{H}_{0}=\sum_{\mathbf{k} \in \mathcal{R}} \omega_{k} \hat{a}_{\mathbf{k}}^{\dagger} \hat{a}_{\mathbf{k}} \\
& \hat{H}_{\mathrm{i}}=\sum_{i=1}^{2} \frac{q_{i}}{\sqrt{2 V}} \sum_{\mathbf{k} \in \mathcal{R}} \frac{1}{\sqrt{\omega_{k}}}\left[\hat{a}_{\mathbf{k}} \tilde{\rho}_{i}^{*}(\mathbf{k}) e^{i \mathbf{k} \cdot \mathbf{y}_{i}}+\hat{a}_{\mathbf{k}}^{\dagger} \tilde{\rho}_{i}(\mathbf{k}) e^{-i \mathbf{k} \cdot \mathbf{y}_{i}}\right],
\end{aligned}
$$

where $\tilde{\rho}_{i}$ is the Fourier transform of $\rho_{i}$

$$
\tilde{\rho}_{i}(\mathbf{k})=\int_{\mathscr{P}} \rho_{i}(\mathbf{x}) e^{-i \mathbf{k} \cdot \mathbf{x}} d^{3} x .
$$

Note that $\tilde{\rho}_{i}^{*}(\mathbf{k})=\tilde{\rho}_{i}(-\mathbf{k})$ as a consequence of $\rho_{i}$ being real, and that if we take $\rho_{i}$ to be spherically symmetric then $\tilde{\rho}_{i}$ depends only on the modulus $k$.

Introducing the operators $\hat{q}_{\mathbf{k}}$ and $\hat{p}_{\mathbf{k}}$ as follows

$$
\hat{q}_{\mathbf{k}}=\frac{1}{\sqrt{2}}\left(\hat{a}_{\mathbf{k}}+\hat{a}_{\mathbf{k}}^{\dagger}\right) \quad \hat{p}_{\mathbf{k}}=\frac{i}{\sqrt{2}}\left(\hat{a}_{\mathbf{k}}^{\dagger}-\hat{a}_{\mathbf{k}}\right)
$$


which therefore obey the CCR

$$
\left[\hat{q}_{\mathbf{k}}, \hat{q}_{\mathbf{k}^{\prime}}\right]=\left[\hat{p}_{\mathbf{k}}, \hat{p}_{\mathbf{k}^{\prime}}\right]=0, \quad\left[\hat{q}_{\mathbf{k}}, \hat{p}_{\mathbf{k}^{\prime}}\right]=i \delta_{\mathbf{k}, \mathbf{k}^{\prime}} \quad,
$$

we obtain the following expression for the total Hamiltonian

$$
\hat{H}=B+C+\frac{1}{2} \sum_{\mathbf{k} \in \mathcal{R}} \omega_{k}\left[\left(\hat{p}_{\mathbf{k}}+\sigma_{\mathbf{k}}\right)^{2}+\left(\hat{q}_{\mathbf{k}}+\tau_{\mathbf{k}}\right)^{2}-1\right],
$$

where

$$
\tau_{\mathbf{k}}=\frac{g}{\sqrt{V \omega_{k}^{3}}} \sum_{i=1}^{2} q_{i} C_{\mathbf{k}}^{i} \quad \sigma_{\mathbf{k}}=-\frac{g}{\sqrt{V \omega_{k}^{3}}} \sum_{i=1}^{2} q_{i} S_{\mathbf{k}}^{i}
$$

with $^{1}$

$$
\begin{aligned}
& C_{\mathbf{k}}^{i}=\Re\left[\tilde{\rho}_{i}(\mathbf{k})\right] \cos \left(\mathbf{k} \cdot \mathbf{y}_{i}\right)+\Im\left[\tilde{\rho}_{i}(\mathbf{k})\right] \sin \left(\mathbf{k} \cdot \mathbf{y}_{i}\right) \\
& S_{\mathbf{k}}^{i}=-\Im\left[\tilde{\rho}_{i}(\mathbf{k})\right] \cos \left(\mathbf{k} \cdot \mathbf{y}_{i}\right)+\Re\left[\tilde{\rho}_{i}(\mathbf{k})\right] \sin \left(\mathbf{k} \cdot \mathbf{y}_{i}\right)
\end{aligned}
$$

The numbers $B$ and $C$ satisfy

$$
B+C=-\frac{1}{2} \sum_{\mathbf{k} \in \mathcal{R}} \omega_{k}\left(\sigma_{\mathbf{k}}^{2}+\tau_{\mathbf{k}}^{2}\right)
$$

and indicating $g_{i}=g q_{i}$ they explicitly read

$$
\begin{gathered}
\begin{aligned}
B=-\frac{g_{1} g_{2}}{V} \sum_{\mathbf{k} \in \mathcal{R}} \frac{1}{\omega_{k}^{2}}\left\{\Re\left[\tilde{\rho}_{1}(\mathbf{k}) \tilde{\rho}_{2}^{*}(\mathbf{k})\right] \cos \left[\mathbf{k} \cdot\left(\mathbf{y}_{1}-\mathbf{y}_{2}\right)\right]+\right. \\
\left.+\Im\left[\tilde{\rho}_{1}(\mathbf{k}) \tilde{\rho}_{2}^{*}(\mathbf{k})\right] \sin \left[\mathbf{k} \cdot\left(\mathbf{y}_{1}-\mathbf{y}_{2}\right)\right]\right\}
\end{aligned} \\
C=-\sum_{i=1}^{2} \frac{g_{i}^{2}}{2 V} \sum_{\mathbf{k} \in \mathcal{R}} \frac{1}{\omega_{k}^{2}}\left|\tilde{\rho}_{i}(\mathbf{k})\right|^{2}
\end{gathered}
$$

\subsection{The physical meaning of $B$ and $C$}

Comparing these expressions with the corresponding ones in $[4,12]$, it is apparent that the expressions (2.12) and (2.16) for the Hamiltonian have exactly the same form as those for point-like sources. The difference shows only in the explicit expression of $\tau_{\mathbf{k}}$ and $\sigma_{\mathbf{k}}$ in terms of the parameters of the model.

\footnotetext{
${ }^{1} \Re[z]$ and $\Im[z]$ indicate respectively the real and the imaginary part of $z \in \mathbb{C}$.
} 
Regarding $B$ and $C$, their expressions in the point-like case are

$$
\begin{aligned}
B_{\mathrm{pt}} & =-\frac{g_{1} g_{2}}{V} \sum_{\mathbf{k} \in \mathcal{R}} \frac{1}{\omega_{k}^{2}} \cos \left[\mathbf{k} \cdot\left(\mathbf{y}_{1}-\mathbf{y}_{2}\right)\right], \\
C_{\mathrm{pt}} & =-\frac{g_{1}^{2}+g_{2}^{2}}{2 V} \sum_{\mathbf{k} \in \mathcal{R}} \frac{1}{\omega_{k}^{2}}
\end{aligned}
$$

and, comparing with (2.17) and (2.18), it is apparent that giving a finite spatial width to the sources introduces a modulation in the sum over the reciprocal lattice. This modulation depends only on the modulus $k$ when $\rho_{1}$ and $\rho_{2}$ are spherically symmetric. The expressions (2.19) and (2.20) are reproduced by setting $\tilde{\rho}_{1}=\tilde{\rho}_{2}=1$ in (2.17) and (2.18), which is compatible with the idea that "the Fourier transform of a Dirac delta is a constant".

The central observation is that, as we discuss in section A.1 of the appendix, when $\rho_{1}$ and $\rho_{2}$ have compact support the modulations due to $\tilde{\rho}_{1}$ and $\tilde{\rho}_{2}$ in the expressions (2.17) and (2.18) are rapidly decreasing, and so the infinite sums in (2.17) and (2.18) are absolutely convergent. In comparison, in the point-like sources case the infinite sum in (2.20) is divergent, and the sum in (2.19) has to be understood in a suitable way to be considered convergent. The model with spatially distributed sources is therefore non-singular, unlike the point-like description where $C$ diverges and it is necessary to arbitrarily discard it to progress with the analysis. The search for a less unrealistic description of the sources has automatically provided a model where the divergence is regularized.

Let us look more closely at the meaning of $C$, which is somehow a mysterious object in [4]. This is best seen in the infinite volume limit, which is well defined as we prove in section A.2.1 of the appendix. In section A.2.2 we show that in this limit we have

$$
\begin{aligned}
& B \underset{V \rightarrow \infty}{\longrightarrow}-g_{1} g_{2} \iint_{\mathbb{R}^{6}} r_{1}\left(\mathbf{x}-\mathbf{y}_{1}\right) r_{2}\left(\mathbf{z}-\mathbf{y}_{2}\right) \frac{1}{4 \pi} \frac{e^{-m\|\mathbf{x}-\mathbf{z}\|}}{\|\mathbf{x}-\mathbf{z}\|} d^{3} x d^{3} z, \\
& C \underset{V \rightarrow \infty}{\longrightarrow}-\frac{1}{2} \sum_{i=1}^{2} g_{i}^{2} \iint_{\mathbb{R}^{6}} r_{i}(\mathbf{x}) r_{i}(\mathbf{z}) \frac{1}{4 \pi} \frac{e^{-m\|\mathbf{x}-\mathbf{z}\|}}{\|\mathbf{x}-\mathbf{z}\|} d^{3} x d^{3} z,
\end{aligned}
$$

where the auxiliary functions $r_{1}$ and $r_{2}$ are defined as in (A.4) and are closely related to $\rho_{1}$ and $\rho_{2}$. It is apparent that $C$ is nothing else that the total selfinteraction energy of the sources ( $B$ is the sum of the pair-wise interaction energy between different sources, as was already recognized in [4]). Considering distributed sources therefore permits to recognize that the numbers $B$ and $C$ are two aspects of the same physical mechanism, the interaction via the Yukawa potential mediated by the field. If $\rho_{1}$ and $\rho_{2}$ become more and more peaked, the number $C$ grows unboundedly while $B$ doesn't, since 
in the latter case we are coupling charge distributions which are spatially separated. This reveals that the divergence of $C_{\mathrm{pt}}$ is just a spurious artifact due to a too crude description of the internal structure of the sources, and permits to appreciate that treating $B$ and $C$ on different grounds, as done in [4], is artificial.

\subsection{Energy eigenstates and spectrum}

We now turn to the derivation of the exact solutions of the model. In [4] this is achieved by representing the abstract canonical operators $\hat{q}_{\mathbf{k}}$ and $\hat{p}_{\mathbf{k}}$, and therefore the Hamiltonian, as explicit operators on a space of functionals. We follow the same path here. More precisely, we choose a representation of the CCR in which the operators $\hat{q}_{\mathbf{k}}$ are diagonal. The state vectors of the field are represented as functionals $\Phi(\{q\})$, where $\{q\}=\left\{q_{\mathbf{k}}\right\}_{\mathbf{k} \in \mathcal{R}}$ indicates a family of real numbers indexed by the vectors of the reciprocal lattice. The abstract operators $\hat{q}_{\mathbf{k}}$ and $\hat{p}_{\mathbf{k}}$ are realized respectively as the operator which multiply $\Phi(\{q\})$ by $q_{\mathbf{k}}$, and as the derivative operator with respect to $q_{\mathbf{k}}$ multiplied by the imaginary unit.

The (total) Hamiltonian operator is therefore realized as the differential operator

$$
\hat{\mathcal{H}} \Phi=\left\{B+C+\frac{1}{2} \sum_{\mathbf{k} \in \mathcal{R}} \omega_{k}\left[\left(-i \frac{\partial}{\partial q_{\mathbf{k}}}+\sigma_{\mathbf{k}}\right)^{2}+\left(q_{\mathbf{k}}+\tau_{\mathbf{k}}\right)^{2}-1\right]\right\} \Phi,
$$

whose eigenfunctionals are the infinite products

$$
\Phi_{\{n\}}(\{q\})=\prod_{\mathbf{k} \in \mathcal{R}} \varphi_{n(\mathbf{k})}^{\mathbf{k}}\left(q_{\mathbf{k}}\right),
$$

where the functions $\varphi_{n}^{\mathbf{k}}$ are defined by

$$
\varphi_{n}^{\mathbf{k}}\left(q_{\mathbf{k}}\right)=e^{-i \sigma_{\mathbf{k}} q_{\mathbf{k}}} \psi_{n}\left(q_{\mathbf{k}}+\tau_{\mathbf{k}}\right),
$$

and $\psi_{n}$ is the standard Hermite function of $n$-th degree

$$
\psi_{n}(x)=\frac{(-1)^{n}}{\sqrt{2^{n} n ! \sqrt{\pi}}} e^{\frac{x^{2}}{2}} \frac{d^{n}}{d x^{n}} e^{-x^{2}} .
$$

The energy eigenvalue associated to $\Phi_{\{n\}}$ is

$$
E_{\{n\}}=B+C+\sum_{\mathbf{k} \in \mathcal{R}} n(\mathbf{k}) \omega_{k}
$$

so the energy spectrum is basically the same as that of a free relativistic neutral scalar field of mass $m$, with the only difference of being rigidly 
shifted by the Yukawa-like interaction energy $B+C$ of the fixed charge distributions.

Formally, the only change in the expressions (2.23)-(2.27) with respect to the corresponding equations in $[4,12]$ is that $C$ is now included in $\hat{\mathcal{H}}$ and therefore in its spectrum, and not discarded anymore. Apart from this, the influence of $\rho_{1}$ and $\rho_{2}$ is felt only through the phase and shift factors $\sigma_{\mathbf{k}}$ and $\tau_{\mathbf{k}} \cdot$

\section{The orthogonality of the spaces of state vectors}

From the analysis of the previous section it follows that each family of nonnegative integers $\{n\}=\{n(\mathbf{k})\}_{\mathbf{k} \in \mathcal{R}}$ individuates an energy eigenstate $\Phi_{\{n\}}$. To define the space of state vectors it is then necessary to decide which class of families is associated to physically acceptable states. In [4], this problem is settled by noting that the only energy eigenstates which can be excited from the ground state using a finite amount of energy are those whose associated sequence possesses a finite number of non-zero elements. Therefore, the space of state vectors is defined as the Hilbert space generated by the stationary states of finite energy, that is by the $\Phi_{\{n\}}$ for which the non-zero $n(\mathbf{k})$ are in a finite number. This reasoning holds equally well in our case, so we adopt the same definition of space of state vectors. It follows that, as in [4], the latter has the structure of non-separable Hilbert space of the infinite direct product type, as introduced by von Neumann [3].

\subsection{Disappearance of the orthogonality}

A central result of [4] is that the spaces of state vectors relative to different parameter choices are orthogonal. We now want to investigate whether this result continues to hold in the model with spatially distributed sources. Before discussing the details, it is worthwhile to clarify that, since the model is invariant with respect to the simultaneous rescaling $g \rightarrow c g, q_{1} \rightarrow q_{1} / c$ and $q_{2} \rightarrow q_{2} / c$ by a real number $c$, it is necessary to work here with the products $g_{1}=g q_{1}$ and $g_{2}=g q_{2}$ to avoid this degeneracy undermining our conclusions. So the parameters of the model are: the product of the coupling constant and the total charges $g_{1}, g_{2}$; the position of the charges $\mathbf{y}_{1}, \mathbf{y}_{2}$; the distributions $\rho_{1}, \rho_{2}$.

Be then $\Gamma=\left\{\left(g_{1}, \mathbf{y}_{1}, \rho_{1}\right),\left(g_{2}, \mathbf{y}_{2}, \rho_{2}\right)\right\}$ and $\bar{\Gamma}=\left\{\left(\bar{g}_{1}, \mathbf{z}_{1}, \zeta_{1}\right),\left(\bar{g}_{2}, \mathbf{z}_{2}, \zeta_{2}\right)\right\}$ two choices for the parameters of the model, with $\mathbf{y}_{1} \neq \mathbf{y}_{2}, \mathbf{z}_{1} \neq \mathbf{z}_{2}$ and $\rho_{1}, \rho_{2}, \zeta_{1}, \zeta_{2}$ all compactly supported. As shown in [4, 12], the spaces of state vectors relative to different parameters choices are orthogonal if and only if the corresponding ground states are orthogonal. Let us therefore assume $\Gamma \neq \bar{\Gamma}$, and indicate with $\Phi_{\{0\}}$ the ground state when the parameters choice is $\Gamma$, while $\bar{\Phi}_{\{0\}}$ indicates the ground state when the parameters choice is $\bar{\Gamma}$. 
The calculation of the scalar product between $\bar{\Phi}_{\{0\}}$ and $\Phi_{\{0\}}$ is formally the same as in $[4,12]$, so we get

$$
\left|\left\langle\bar{\Phi}_{\{0\}}, \Phi_{\{0\}}\right\rangle\right|=\prod_{\mathbf{k} \in \mathcal{R}}\left|\left\langle\bar{\varphi}_{0}^{\mathbf{k}}, \varphi_{0}^{\mathbf{k}}\right\rangle\right|=\exp \left\{-\frac{1}{4} \sum_{\mathbf{k} \in \mathcal{R}}\left[\left(\sigma_{\mathbf{k}}-\bar{\sigma}_{\mathbf{k}}\right)^{2}+\left(\tau_{\mathbf{k}}-\bar{\tau}_{\mathbf{k}}\right)^{2}\right]\right\},
$$

and inserting the expressions (2.13)-(2.15) for the phase and shift factors we now find

$$
\begin{aligned}
\left(\sigma_{\mathbf{k}}-\bar{\sigma}_{\mathbf{k}}\right)^{2}+\left(\tau_{\mathbf{k}}-\bar{\tau}_{\mathbf{k}}\right)^{2} \leq \frac{\sqrt{2}}{V \omega_{k}^{3}}\left\{\left[\left|g_{1} \tilde{\rho}_{1}(\mathbf{k})\right|+\left|g_{2} \tilde{\rho}_{2}(\mathbf{k})\right|\right]^{2}+\right. \\
\left.+2 \sum_{i, j=1}^{2}\left|g_{i} \tilde{\rho}_{i}(\mathbf{k})\right|\left|\bar{g}_{j} \tilde{\zeta}_{j}(\mathbf{k})\right|+\left[\left|\bar{g}_{1} \tilde{\zeta}_{1}(\mathbf{k})\right|+\left|\bar{g}_{2} \tilde{\zeta}_{2}(\mathbf{k})\right|\right]^{2}\right\}
\end{aligned}
$$

Since $\rho_{1}, \rho_{2}, \zeta_{1}$ and $\zeta_{2}$ are all compactly supported, we can use exactly the same arguments of section A.1 of the appendix to infer that the infinite sum

$$
\begin{aligned}
\frac{\sqrt{2}}{V} \sum_{\mathbf{k} \in \mathcal{R}} \frac{1}{\omega_{k}^{3}}\left\{\left[\left|g_{1} \tilde{\rho}_{1}(\mathbf{k})\right|+\left|g_{2} \tilde{\rho}_{2}(\mathbf{k})\right|\right]^{2}+\right. \\
\left.+2 \sum_{i, j=1}^{2}\left|g_{i} \tilde{\rho}_{i}(\mathbf{k})\right|\left|\bar{g}_{j} \tilde{\zeta}_{j}(\mathbf{k})\right|+\left[\left|\bar{g}_{1} \tilde{\zeta}_{1}(\mathbf{k})\right|+\left|\bar{g}_{2} \tilde{\zeta}_{2}(\mathbf{k})\right|\right]^{2}\right\}
\end{aligned}
$$

converges (here we even have $\omega^{3}$ instead of $\omega^{2}$ in the denominator). It follows that $\sum_{\mathbf{k} \in \mathcal{R}}\left[\left(\sigma_{\mathbf{k}}-\bar{\sigma}_{\mathbf{k}}\right)^{2}+\left(\tau_{\mathbf{k}}-\bar{\tau}_{\mathbf{k}}\right)^{2}\right]$ converges (absolutely), which in turn implies that

$$
\left|\left\langle\bar{\Phi}_{\{0\}}, \Phi_{\{0\}}\right\rangle\right| \neq 0
$$

Therefore we conclude that, when the sources are spatially distributed, the spaces of state vectors relative to different parameter choices are never orthogonal, no matter how peaked the distributions. This result is compatible with the exposition of [14] regarding a relativistic and massive scalar quantum field interacting with an external potential, although in the latter case the source is assumed to be of compact support also in the time variable.

\subsection{Divergence $v s$ orthogonality}

To be more specific, let us focus on the case where $\bar{g}_{1}=\bar{g}_{2}=0$, that is comparing the energy eigenstates associated to the parameters choice $\Gamma=$ $\left\{\left(g_{1}, \mathbf{y}_{1}, \rho_{1}\right),\left(g_{2}, \mathbf{y}_{2}, \rho_{2}\right)\right\}$ with the energy eigenstates of the free Hamiltonian. In this case we have $\bar{\tau}_{\mathbf{k}}=\bar{\sigma}_{\mathbf{k}}=0$ so from the previous section we get

$$
\left|\left\langle\Phi_{\{0\}}^{\text {free }}, \Phi_{\{0\}}\right\rangle\right|=\exp \left[-\frac{1}{4} \sum_{\mathbf{k} \in \mathcal{R}}\left(\sigma_{\mathbf{k}}^{2}+\tau_{\mathbf{k}}^{2}\right)\right],
$$


which implies that the space of state vectors labeled by $\Gamma$ and the space of free state vectors are orthogonal if and only if

$$
\sum_{\mathbf{k} \in \mathcal{R}}\left(\sigma_{\mathbf{k}}^{2}+\tau_{\mathbf{k}}^{2}\right)=\infty .
$$

On the other hand, recalling from (2.16) that

$$
B+C=-\frac{1}{2} \sum_{\mathbf{k} \in \mathcal{R}} \omega_{k}\left(\sigma_{\mathbf{k}}^{2}+\tau_{\mathbf{k}}^{2}\right)
$$

and comparing the latter with (3.5), we note that the condition of the ground state energy $B+C$ being divergent is closely related to the condition of orthogonality of the free and interacting spaces of state vectors, being just slightly weaker (due to the factor $\omega_{k}$ ).

This implies that it is not possible to achieve the phenomenon of orthogonality of the free and interacting spaces of state vectors with a well-defined model, that is one where $B+C$ is finite. This follows from the fact that if (3.5) holds, then surely (3.6) diverges. On the other hand, it may be possible to have a model which is singular and yet where the free and interacting spaces of state vectors are not orthogonal. For the sake of completeness, let us look for simple conditions for this (admittedly not very interesting) possibility to happen. Let us assume that the two functions $\rho_{1}$ and $\rho_{2}$ are equal $(=\rho)$ and spherically symmetric, and work in the infinite volume limit. Recalling from (2.13)-(2.15) that in this case

$$
\sigma_{\mathbf{k}}^{2}+\tau_{\mathbf{k}}^{2}=\frac{|\tilde{\rho}(k)|^{2}}{V \omega_{k}^{3}}\left\{g_{1}^{2}+g_{2}^{2}+2 g_{1} g_{2} \cos \left[\mathbf{k} \cdot\left(\mathbf{y}_{1}-\mathbf{y}_{2}\right)\right]\right\}
$$

any $\tilde{\rho}$ such that

$$
0 \neq \lim _{k \rightarrow \infty} k^{\beta}|\tilde{\rho}(k)|^{2}<\infty
$$

for some $\beta$ in the interval $0<\beta \leq 1$ satisfies the condition of $B+C$ diverging while the infinite sum in (3.5) being finite. In this case $\tilde{\rho}$ is not even square-integrable. In particular, the same results we obtained for compactly supported distributions would hold also if, in the infinite volume limit, we considered $\rho_{1}$ and $\rho_{2}$ to be rapidly decreasing (for example taking them equal to the electronic distribution of the ground state of the hydrogen atom).

\subsubsection{Comparison with the point-like case}

It is interesting to see explicitly how the orthogonality result of [4] is recovered in our description. Going back to the general case $\bar{g}_{1} \neq 0, \bar{g}_{2} \neq 0$, let us consider for simplicity the case where the distributions are all equal, that 
is $\rho_{1}=\rho_{2}=\zeta_{1}=\zeta_{2}=\rho$. We obtain

$$
\begin{aligned}
\sum_{\mathbf{k} \in \mathcal{R}} & {\left[\left(\sigma_{\mathbf{k}}-\bar{\sigma}_{\mathbf{k}}\right)^{2}+\left(\tau_{\mathbf{k}}-\bar{\tau}_{\mathbf{k}}\right)^{2}\right]=} \\
= & \frac{1}{V} \sum_{\mathbf{k} \in \mathcal{R}} \frac{|\tilde{\rho}(\mathbf{k})|^{2}}{\omega_{k}^{3}}\left\{g_{1}^{2}+g_{2}^{2}+\bar{g}_{1}^{2}+\bar{g}_{2}^{2}+2 g_{1} g_{2} \cos \left[\mathbf{k} \cdot\left(\mathbf{y}_{1}-\mathbf{y}_{2}\right)\right]+\right. \\
& \left.+2 \bar{g}_{1} \bar{g}_{2} \cos \left[\mathbf{k} \cdot\left(\mathbf{z}_{1}-\mathbf{z}_{2}\right)\right]-2 \sum_{i, j=1}^{2} g_{i} \bar{g}_{j} \cos \left[\mathbf{k} \cdot\left(\mathbf{y}_{i}-\mathbf{z}_{j}\right)\right]\right\}
\end{aligned}
$$

which again converges absolutely since $\tilde{\rho}$ is rapidly decreasing. The corresponding result of $[4,12]$ can be retrieved by setting $|\tilde{\rho}(\mathbf{k})|=1$. In this case the denominator $\omega_{k}^{3}$ is not sufficient by itself to make the infinite sum converge, and for the convergence to take place suitable cancellations need to happen inside the round parenthesis in the right hand side (even in this case, though, the infinite sum does not converges absolutely and we need to define the order of summation in a suitable way to be able to consider the sum convergent). The condition for such cancellations to happen turns out to be $\Gamma=\bar{\Gamma}$, which is the celebrated result of [4].

\subsubsection{Algebraic treatment}

It is worthwhile to remind that the orthogonality condition (3.5) can be also derived by algebraic means. Following [7], we introduce the operators

$$
\begin{aligned}
& \hat{A}_{\mathbf{k}}=\frac{1}{\sqrt{2}}\left[\hat{q}_{\mathbf{k}}+\tau_{\mathbf{k}}+i\left(\hat{p}_{\mathbf{k}}+\sigma_{\mathbf{k}}\right)\right]=\hat{a}_{\mathbf{k}}+\alpha_{\mathbf{k}} \\
& \hat{A}_{\mathbf{k}}^{\dagger}=\frac{1}{\sqrt{2}}\left[\hat{q}_{\mathbf{k}}+\tau_{\mathbf{k}}-i\left(\hat{p}_{\mathbf{k}}+\sigma_{\mathbf{k}}\right)\right]=\hat{a}_{\mathbf{k}}^{\dagger}+\alpha_{\mathbf{k}}^{*}
\end{aligned}
$$

where $\alpha_{\mathbf{k}}=\tau_{\mathbf{k}}+i \sigma_{\mathbf{k}}$. Since the commutation rules for $\hat{A}_{\mathbf{k}}$ and $\hat{A}_{\mathbf{k}}^{\dagger}$ are equal to those for $\hat{a}_{\mathbf{k}}$ and $\hat{a}_{\mathbf{k}}^{\dagger}$, to wit (2.7), the total (interacting) Hamiltonian can be written as

$$
\hat{H}=B+C+\sum_{\mathbf{k} \in \mathcal{R}} \omega_{k} \hat{A}_{\mathbf{k}}^{\dagger} \hat{A}_{\mathbf{k}}
$$

so its structure is formally similar to that of the free Hamiltonian.

Coherently with the discussion of section 2.4, the operators $\hat{A}$ and $\hat{A}^{\dagger}$ are defined on a non-separable Hilbert space $\mathscr{H}$ of the infinite direct product type. Let us realize the non-constant part of the Hamiltonian $\hat{H}$ as the limit of a sequence of operators indexed by $n \in \mathbb{N}$, such that the $n$-th element of the sequence reads

$$
\sum_{\mathbf{k} \in \mathcal{R},\|\mathbf{k}\| \leq n} \omega_{k} \hat{A}_{\mathbf{k}}^{\dagger} \hat{A}_{\mathbf{k}}
$$


Although the sequence of operators is defined on (a dense subset of) $\mathscr{H}$, the limit as $n \rightarrow \infty$ of this sequence converges only on a (dense subset of a) separable subspace $\mathcal{H}_{\Gamma} \subset \mathscr{H}$, which in general depends on the choice of the parameters collectively indicated with $\Gamma$ [7]. Wightman and Schweber assert that, if

$$
\sum_{\mathbf{k} \in \mathcal{R}}\left|\alpha_{\mathbf{k}}\right|^{2}<\infty
$$

then all the subspaces $\mathcal{H}_{\Gamma}$ coincide, that is $\mathcal{H}_{\Gamma}=\mathcal{H}_{\bar{\Gamma}}$ even if $\Gamma \neq \bar{\Gamma}$. On the other hand, if

$$
\sum_{\mathbf{k} \in \mathcal{R}}\left|\alpha_{\mathbf{k}}\right|^{2}=\infty
$$

then the subspaces $\mathcal{H}_{\Gamma}$ corresponding to different parameters choices are orthogonal, that is $\Gamma \neq \bar{\Gamma}$ implies $\mathcal{H}_{\Gamma} \perp \mathcal{H}_{\bar{\Gamma}}$. The connection with the previous analysis, and in particular with the condition (3.5), becomes evident once we recognize that

$$
\left|\alpha_{\mathbf{k}}\right|^{2}=\sigma_{\mathbf{k}}^{2}+\tau_{\mathbf{k}}^{2}
$$

and that (3.4) can be expressed as

$$
\left|\left\langle\Phi_{\{0\}}^{\text {free }}, \Phi_{\{0\}}\right\rangle\right|=\exp \left(-\frac{1}{4} \sum_{\mathbf{k} \in \mathcal{R}}\left|\alpha_{\mathbf{k}}\right|^{2}\right) .
$$

\section{Discussion}

The results of the previous sections unambiguously show that the divergence of the ground state energy $B+C$ of the model is strongly correlated with the property of orthogonality of the spaces of state vectors. The common underlying cause of both is the non convergence of the infinite sum $\sum_{\mathbf{k} \in \mathcal{R}}\left(\sigma_{\mathbf{k}}^{2}+\tau_{\mathbf{k}}^{2}\right)$. The two conditions are strictly speaking not equivalent, but the important point for our purposes is that it is impossible to have orthogonality while having a finite ground state energy.

This prompts a revision of the validity of Van Hove's results. It is useful in this sense to identify two logically distinct parts in Van Hove's article. The first is the derivation, from the toy model of a scalar field interacting linearly with point-like sources, of the quantum Hamiltonian

$$
\begin{aligned}
& \hat{H}_{\mathrm{pt}}=B+\frac{1}{2} \sum_{\mathbf{k} \in \mathcal{R}} \omega_{k}\left[\left(\hat{p}_{\mathbf{k}}+\sigma_{\mathbf{k}}\right)^{2}+\left(\hat{q}_{\mathbf{k}}+\tau_{\mathbf{k}}\right)^{2}-1\right], \\
& \sum_{\mathbf{k} \in \mathcal{R}}\left(\sigma_{\mathbf{k}}^{2}+\tau_{\mathbf{k}}^{2}\right)=\infty
\end{aligned}
$$

the condition (4.1b) being a defining property of the Hamiltonian. Note that $C$ is discarded, while $B$, which is finite, is kept. The second part is 
the study of this Hamiltonian and its phenomenology. The validity of both parts contribute to the relevance of the article as a whole. We discuss below the consequence of our results for the validity of each of these two parts. To avoid cumbersome phrases, below we use the term "Van Hove's model" to indicate the model of a scalar field interacting linearly with point-like, infinitely massive sources, without a priori identifying it with the Hamiltonian (4.1a)-(4.1b).

\subsection{The model and the quantum Hamiltonian}

\subsubsection{On neglecting infinities}

One possible justification for associating the quantum Hamiltonian (4.1a)(4.1b) to Van Hove's model is the assertion that it is a common practice to neglect infinities in QFT. It could in fact be argued that, since the ground state energy of the oscillators is neglected right after quantizing, it should be safe to neglect any other infinite contribution to the ground state energy of the system. From this point of view, the question seems to boil down to whether and when it is justified to "neglect infinities". It cannot be avoided to note how the fact that neglecting infinities has become routine in modern physics is playing here a confounding role.

The case of $C$ and that of the infinite energy contribution coming from the ground state of the oscillators are, however, qualitatively different. The latter is of kinematical character: it is present whenever a scalar field is quantized and is independent of the dynamics of the specific model under consideration (i.e. from the interaction Hamiltonian). Specifically, in our case the partial sums associated to the diverging series are independent of the value of the coupling constant and of the position of the charges. On the other hand, the contribution from $C$ has a dynamical character: the partial sums associated to the diverging series depend on the coupling constant and on the distribution of the charges. This is a signal that the divergence of $C$ may be pointing to a relevant dynamical mechanism which is not being taken into account appropriately.

We believe that the discussion in section 2.3 makes a strong point: $B$ and $C$ are not different in essence, and both have an important physical meaning. Therefore the divergence of $C$ is not to be seen as something which can be lightly discarded, on the contrary it signals that the original model, with point-like sources, is ill-defined.

\subsubsection{On treating $B$ and $C$ differently}

To exemplify how artificial it is to treat $B$ and $C$ on different grounds, it is useful to reconsider the discussion in [4] (recalled in section 5.3 of [12]) about the applicability of the perturbative approach to evaluate the interaction 
energy $B$ between different sources, and the scalar product between the free and the interacting ground state.

In the first case, the second order perturbative correction to the interaction energy is evaluated imposing a momentum cut-off, "excluding every transition where one boson is emitted and absorbed by the same source" (in Van Hove's words). The result indeed remains finite when removing the cutoff, causing Van Hove to conclude that the perturbative method is applicable to estimate $B$. However, the total second order perturbative correction to the ground state energy explicitly reads

$$
E_{\{0\}}^{(2)}(\Lambda)=-g^{2} \frac{q_{1}^{2}+q_{2}^{2}}{2 V} \sum_{\mathbf{k} \in \mathcal{R}_{\Lambda}} \frac{1}{\omega_{k}^{2}}-g^{2} \frac{q_{1} q_{2}}{V} \sum_{\mathbf{k} \in \mathcal{R}_{\Lambda}} \frac{\cos \left[\mathbf{k} \cdot\left(\mathbf{y}_{1}-\mathbf{y}_{2}\right)\right]}{\omega_{k}^{2}},
$$

where $\Lambda$ is the cut-off and $\mathcal{R}_{\Lambda}$ indicates the set of vectors of the reciprocal lattice of modulus $\leq \Lambda$. Therefore, what Van Hove is really doing is to discard the addend multiplied by $q_{1}^{2}+q_{2}^{2}$, which after removing the cut-off would give rise to the number $C$. This is the term which corresponds to "bosons being emitted and absorbed by the same source" (more precisely, the contribution associated to $q_{i}^{2}$ corresponds to "bosons being emitted and absorbed by the source of charge $\left.q_{i}{ }^{\prime \prime}\right)$. Only the addend multiplied by the mixed product $q_{1} q_{2}$ is kept, which after removing the cut-off $(\Lambda \rightarrow \infty)$ gives rise to $B$ and remains finite (if the summation is understood appropriately).

Consider now the second case, where the (absolute value of the) scalar product $a$ between the free and the interacting ground state is considered. Imposing again a momentum cut-off $\Lambda$ and working at the second order in perturbations, the result

$$
a^{(2)}(\Lambda)=1-\frac{g^{2}}{4 V} \sum_{\mathbf{k} \in \mathcal{R}_{\Lambda}} \frac{1}{\omega_{k}^{3}}\left\{q_{1}^{2}+q_{2}^{2}+2 q_{1} q_{2} \cos \left[\mathbf{k} \cdot\left(\mathbf{y}_{1}-\mathbf{y}_{2}\right)\right]\right\}
$$

displays again the sum of two terms, one containing $q_{1}^{2}+q_{2}^{2}$ and the other containing $q_{1} q_{2}$. This time the former term is not neglected, reaching the conclusion that the perturbative approach for $a$ is unreliable. Crucially, however, the divergence is entirely due to the $q_{1}^{2}+q_{2}^{2}$ term: if we neglected it, we would remain with

$$
a^{(2)}(\Lambda)=1-g^{2} \frac{q_{1} q_{2}}{2 V} \sum_{\mathbf{k} \in \mathcal{R}_{\Lambda}} \frac{\cos \left[\mathbf{k} \cdot\left(\mathbf{y}_{1}-\mathbf{y}_{2}\right)\right]}{\omega_{k}^{3}},
$$

which has a finite limit when we remove the cut-off (again, if the summation is understood suitably, since the convergence is not absolute). The conclusion that evaluating $a$ with the perturbative approach we would get a series of the form $1-g^{2} \cdot \infty+g^{4} \cdot \infty-\ldots$ is therefore entirely based on the decision 
to keep the $q_{1}^{2}+q_{2}^{2}$ term. Note that a finite limit of $a^{(2)}$ when removing the cut-off would mean that the free and interacting spaces of state vectors are not orthogonal.

It seems to us that the disparity is striking: the same type of term is neglected in one case and kept in the other, and this choice determines the finiteness or the divergence after removing the cut-off. Neglecting the $q_{1}^{2}+q_{2}^{2}$ term in the first case implements the act of treating $B$ and $C$ differently. If we treated $B$ and $C$ on the same ground, we would have an infinite ground state energy (i.e. an ill-defined model) and orthogonality of the free and interacting spaces of state vectors of the Hamiltonian (4.1a)-(4.1b) (which could not be said, therefore, to describe the model). If we insisted in neglecting $C$, we should at least recognize that the diverging term in the expression for $a$ is related to it, and so for consistency we should neglect it as well. We would then have a finite ground state energy and the orthogonality would disappear. This is exactly the moral that the study of the model with distributed sources teaches.

\subsubsection{The moral on the first part}

All considered, we are of the opinion that the Hamiltonian (4.1a)-(4.1b) cannot be regarded as the quantum description of a scalar field interacting linearly with fixed sources. For, if these source were point-like, then the condition (4.1b) would hold but in this case we should retain the (divergent) $C$ in the Hamiltonian, and so (4.1a) would not be correct. If on the other hand the sources were distributed, we could safely neglect $C$ and work with the Hamiltonian (4.1a) but we should consistently abandon (4.1b) in favor of a converging series.

Therefore, we propose that Van Hove's model can be considered at best as a pictorial inspiration to motivate the interest in the Hamiltonian (4.1a)(4.1b), but that there is no strict connection between the two. Moreover, in light of the strong correlation between the divergence of $C$ and the phenomenon of orthogonality of the spaces of state vectors, we believe that the latter phenomenon cannot be ascribed to a quantum scalar field interacting linearly with sources lying at fixed positions (but just to the abstract Hamiltonian (4.1a)-(4.1b) itself).

\subsection{The quantum Hamiltonian and its relevance}

The phenomenology associated to the Hamiltonian (4.1a)-(4.1b) informs all the discussion in [4] regarding the formal structure of interacting QFT and the use of the perturbative approach in that context. Since this is the core and the most important part of Van Hove's article, the criticism we exposed in the previous section calls into question the relevance of Van Hove's article as a whole (save for its historical interest). Let us briefly assess how Van 
Hove's discussion is affected by our results.

\subsubsection{Van Hove's and our results}

Regarding the formal structure of QFT, the message that Van Hove's article transmits to the contemporary reader is that the use of UIR of the CCR is an important ingredient to describe an interacting QFT. Regarding the perturbative approach, instead, its main argument is that a change in the value of the coupling constant, no matter how small, changes the free space of state vectors into an altogether different space, and this should be taken into account when performing a perturbative analysis (with particular relevance for the interaction picture). Both of these arguments rest on the property of orthogonality of the spaces of state vectors relative to different values of the parameters of the Hamiltonian (4.1a)-(4.1b).

It is fair to say that, if the orthogonality phenomenon were displayed just by an abstract Hamiltonian chosen ad hoc, one may doubt whether this really informs something about a realistic QFT. Conversely, if the Hamiltonian (4.1a)-(4.1b) described the consistent quantization of a concrete (although crude) model of interacting field, the outlook of his results would be more prominent. From this point of view, the lack of strict connection between the Hamiltonian (4.1a)-(4.1b) and Van Hove's model seem to subtract a fair amount of appeal from his article. For, to support the conjecture that the use of UIR of the CCR is important to describe an interacting QFT, it would now be necessary to motivate directly why the Hamiltonian (4.1a)-(4.1b) should be representative of the formal structure of a realistic QFT. This was not done in Van Hove's article, and it is far from obvious why it should be so.

It is however worthwhile to point out that the model itself is very different from, and not easily related to, a realistic interacting QFT. Apart from the crudeness of the approximation on the structure of the sources, it is important to recall that non-linearities are absent in Van Hove's model: the quantum field is not self-interacting, and couples linearly with the sources. So it is far from clear what such a model could really teach about a realistic interacting case. From this point of view, the lack of strict connection between the Hamiltonian (4.1a)-(4.1b) and Van Hove's model seems less tragic.

\subsubsection{The moral on the second part}

Overall, we believe that Van Hove's article retains some strength. From our point of view, its value does not lie in the certainty with which it indicates the mathematical properties which a non-perturbative QFT should have, but in the fact that it points to concepts, nowadays frequently overlooked, which is still worth keeping in mind. There is no certainty that these concepts will 
turn out to be useful. But, they should be known by those who, aiming to go beyond the perturbative renormalization prescription, look for a finite and non-perturbative formulation of a realistic interacting QFT which be less lofty than the algebraic one [15] (if indeed this is possible). Other theoretical research programs may benefit by such a knowledge, see [16] for an example of application in Quantum Gravity.

Indeed Van Hove's article exposes very clearly how UIR of the CCR in a separable Hilbert space can coexist as embedded in an "ambient" nonseparable Hilbert space, the inequivalence being reflected in the orthogonality between distinct separable subspaces. It also shows that the appearance of UIR of the CCR is not to be ascribed just to very contrived and obscure Hamiltonians, but it emerges even from the simple Hamiltonian (4.1a)-(4.1b). Moreover, it exposes these facts in a very clear way since the latter is exactly solvable. These are remarkable merits, which are unscathed by our present analysis.

On another point, it suggests that the very space of state vectors may not be something given once for all, but it may vary with the value of the coupling (more in general, with the Hamiltonian). Being aware of this possibility is important. The identification of the interaction picture as something problematic in QFT, potentially contributing to the appearance of perturbative divergences, is also a valuable insight which chronologically precedes and complements the body of results known under the name of "Haag's theorem".

If, in the intervening years from its publication, the awareness of these concepts had pervaded the physicists community, we could even say that Van Hove's article would retain just a historical interest. Since this has not happened, we are of the opinion that the article is still worth to be read.

\section{Conclusions}

Léon Van Hove in [4] studied a simple model where a scalar quantum field interacts linearly with point-like masses lying at fixed positions. He concluded that the spaces of state vectors relative to different values of the parameters of the Hamiltonian (such as the coupling constant, the number and the position of the sources) are orthogonal one to the other. While acknowledging the crudeness of the model, he conjectured that the spaces of state vectors relative to different values of the coupling constant would remain orthogonal, or at least different, also in a more realistic QFT. This conjecture was put forward despite the presence of a divergence in the exact solutions, which was discarded without any justification and whose physical meaning was not discussed.

We investigated the validity of Van Hove's conjecture by considering a slightly less irrealistic model where the sources still lie at fixed positions, but have finite spatial width. Our results indicate that, whenever the sources 
are modeled in a less irrealistic way, the phenomenon of orthogonality of the spaces of state vectors disappears. Moreover, we uncovered the physical meaning of the above mentioned divergence and showed that it is intimately linked to the orthogonality phenomenon, to the effect that regularizing the divergence kills the orthogonality of the spaces of state vectors.

We suggest that Van Hove's article is still worth reading since it displays, through a very simple Hamiltonian which can be solved exactly, the presence of Unitarily Inequivalent Representations of the Canonical Commutation Relations, which are associated to different values of the parameters of the Hamiltonian. Importantly, it shows how all these representations can coexist in a single non-separable Hilbert space. Moreover, it points out a possible harmful role of the interaction picture in the case where the space of state vectors depends on the coupling. These are potentially useful insights, even for applications beyond pure Quantum Field Theory, that are usually overlooked nowadays.

However, the lack of connection of this Hamiltonian even with a very crude QFT model weakens the authority of the conjecture that the mathematical structures uncovered in the article should necessarily be an important part of the non-perturbative description of a realistic interacting QFT.

It is interesting to point out that, shortly after the appearance of Van Hove's article, an article by A. Loinger appeared [17], in which the quantized dynamics of two strings connected by springs was studied. The phenomenon of orthogonality of the spaces of state vectors was found to be absent in the model, and Van Hove offered a qualitative explanation for this absence which did not contradict the results of his article, as recounted in [18]. In retrospective, it is hard not to wonder whether his argument was really as sound as previously thought.

\section{Acknowledgments}

The author acknowledges partial financial support from the Fundação de Amparo à Pesquisa do Estado do Rio de Janeiro (FAPERJ, Brazil) under the Programa de Apoio à Docência (PAPD) program.

\section{A Results about the infinite sums}

In this appendix we collect results about the infinite sums in (2.17) and (2.18). As we see below, the character of convergence of all these sums can be traced back to that of the infinite sum

$$
\frac{1}{V} \sum_{\mathbf{k} \in \mathcal{R}} \frac{1}{\omega_{k}^{2}}|\tilde{\rho}(\mathbf{k})|^{2},
$$


where $\tilde{\rho}(\mathbf{k})$ is the Fourier coefficient of a function $\rho$ periodic on the cubic lattice and compactly supported in its primitive cell $\mathscr{P}$.

A word about the notation. In what follows, a triple $\alpha=\left(\alpha_{1}, \alpha_{2}, \alpha_{3}\right)$ of non-negative integers is called a tri-index, and the space of tri-indices is indicated with $I_{+}^{3}$. We indicate

$$
\mathbf{x}^{\alpha}=x_{1}^{\alpha_{1}} x_{2}^{\alpha_{2}} x_{3}^{\alpha_{3}}, \quad \partial^{\beta}=\partial_{1}^{\beta_{1}} \partial_{2}^{\beta_{2}} \partial_{3}^{\beta_{3}} .
$$

We moreover indicate with $C_{c}^{\infty}$ the set of smooth functions with compact support, and $\mathscr{S}$ indicates the Schwartz space of rapidly decreasing functions which by definition satisfy

$$
\sup _{\mathbf{x} \in \mathbb{R}^{3}}\left|\mathbf{x}^{\alpha} \partial^{\beta} f\right|<\infty \quad, \quad \forall \alpha, \beta \in I_{+}^{3}
$$

\section{A.1 Convergence}

To understand the behavior of $\tilde{\rho}(\mathbf{k})$ when $k \rightarrow \infty$, it is useful to introduce an auxiliary function $r$ closely related to $\rho$. While the latter is periodic on the lattice, and it can be thought as being defined on the primitive cell $\mathscr{P}$ and extended by periodicity, let us consider a non-periodic function $r: \mathbb{R}^{3} \rightarrow \mathbb{R}$ which coincides with $\rho$ only on the primitive cell, that is

$$
r(\mathbf{x})= \begin{cases}\rho(\mathbf{x}) & \text { if } \mathbf{x} \in \mathscr{P} \\ 0 & \text { elsewhere }\end{cases}
$$

Clearly, $r$ belongs to $C_{c}^{\infty}\left(\mathbb{R}^{3}\right)$ as a consequence of $\rho$ belonging to $C_{c}^{\infty}(\mathscr{P})$. Defining the Fourier transform of $r$ as

$$
\tilde{r}(\mathbf{k})=\int_{\mathbb{R}^{3}} r(\mathbf{x}) e^{-i \mathbf{k} \cdot \mathbf{x}} d^{3} x,
$$

the comparison of (2.9) with (A.5) reveals that, by the way we defined $r$, we have

$$
\tilde{r}(\mathbf{k})=\tilde{\rho}(\mathbf{k}) \quad, \quad \forall \mathbf{k} \in \mathcal{R} \quad .
$$

In other words, the Fourier transform of $r$ evaluated at a vector $\mathbf{k}$ of the reciprocal lattice coincides with the Fourier coefficient of $\rho$ evaluated at the same $\mathbf{k}$. With this in mind, it is possible to work with $\tilde{r}$ instead of with $\tilde{\rho}$ whenever convenient.

Recall now that the Fourier transform, as an operator, is a bijection of $\mathscr{S}\left(\mathbb{R}^{3}\right)$ onto $\mathscr{S}\left(\mathbb{R}^{3}\right)$ [19]. Since $C_{c}^{\infty}\left(\mathbb{R}^{3}\right) \subset \mathscr{S}\left(\mathbb{R}^{3}\right)$ it follows that $r \in \mathscr{S}\left(\mathbb{R}^{3}\right)$, and therefore $\tilde{r} \in \mathscr{S}\left(\mathbb{R}^{3}\right)$ as well. By the property (A.3), the fact that $\tilde{r}$ is rapidly decreasing implies that for every $\alpha \in I_{+}^{3}$ we have

$$
\left.\sup _{\mathbf{k} \in \mathbb{R}^{3}}\left|\mathbf{k}^{\alpha} \frac{1}{\omega_{k}^{2}}\right| \tilde{r}(\mathbf{k})\right|^{2} \mid<\infty
$$


and taking into account (A.6) we get

$$
\left.\sup _{\mathbf{k} \in \mathcal{R}}\left|\mathbf{k}^{\alpha} \frac{1}{\omega_{k}^{2}}\right| \tilde{\rho}(\mathbf{k})\right|^{2} \mid<\infty \quad,
$$

again for every $\alpha \in I_{+}^{3}$. In particular, $|\tilde{\rho}(\mathbf{k})|^{2} / \omega_{k}^{2}$ goes to zero more rapidly than any non-negative power of $1 / k$. Since the number of points of the reciprocal lattice contained in a sphere of radius $k$ diverge as a power of $k$ when $k \rightarrow \infty$, it follows that the infinite sum (A.1) converges absolutely.

This result readily applies to the convergence of the infinite sums in (2.18), since both $\rho_{1}$ and $\rho_{2}$ are compactly supported. Regarding (2.17), note that

$$
\begin{gathered}
\left|\Re\left[\tilde{\rho}_{1}(\mathbf{k}) \tilde{\rho}_{2}^{*}(\mathbf{k})\right] \cos \left[\mathbf{k} \cdot\left(\mathbf{y}_{1}-\mathbf{y}_{2}\right)\right]+\Im\left[\tilde{\rho}_{1}(\mathbf{k}) \tilde{\rho}_{2}^{*}(\mathbf{k})\right] \sin \left[\mathbf{k} \cdot\left(\mathbf{y}_{1}-\mathbf{y}_{2}\right)\right]\right| \leq \\
\leq\left|\Re\left[\tilde{\rho}_{1}(\mathbf{k}) \tilde{\rho}_{2}^{*}(\mathbf{k})\right]\right|+\left|\Im\left[\tilde{\rho}_{1}(\mathbf{k}) \tilde{\rho}_{2}^{*}(\mathbf{k})\right]\right| \leq 2\left|\tilde{\rho}_{1}(\mathbf{k})\right|\left|\tilde{\rho}_{2}(\mathbf{k})\right| \quad, \quad \text { (A.9) }
\end{gathered}
$$

and recall that the product of two rapidly decreasing functions is rapidly decreasing. It follows that the infinite sum in (2.17) converges absolutely.

\section{A.2 Infinite volume limit}

We now consider the infinite volume limit

$$
\lim _{V \rightarrow \infty} \frac{1}{V} \sum_{\mathbf{k} \in \mathcal{R}} \frac{1}{\omega_{k}^{2}}|\tilde{\rho}(\mathbf{k})|^{2}
$$

\section{A.2.1 Existence}

Note first of all that

$$
\frac{1}{V} \sum_{\mathbf{k} \in \mathcal{R}} \frac{1}{\omega_{k}^{2}}|\tilde{\rho}(\mathbf{k})|^{2}=\frac{1}{(2 \pi)^{3}} \sum_{\mathbf{k} \in \mathcal{R}}\left(\frac{2 \pi}{L}\right)^{3} \frac{1}{\omega_{k}^{2}}|\tilde{r}(\mathbf{k})|^{2},
$$

and that the infinite sum on the right hand side is a Riemann sum of the integral

$$
\int_{\mathbb{R}^{3}} \frac{1}{\omega_{k}^{2}}|\tilde{r}(\mathbf{k})|^{2} d^{3} k
$$

Specifically, the cells of the reciprocal lattice are the 3-intervals of the Riemann sum.

The convergence of the integral (A.12) is easy to establish using the results of the previous section. In fact, writing the integral as the limit for 
$K \rightarrow \infty$ of the related integral on the sphere $\mathcal{B}(K)$ of radius $K$ centered at the origin, we have

$$
\lim _{K \rightarrow \infty} \int_{\mathcal{B}(K)} \frac{1}{\omega_{k}^{2}}|\tilde{r}(\mathbf{k})|^{2} d^{3} k \leq \lim _{K \rightarrow \infty} \int_{0}^{K} \frac{4 \pi k^{2}}{\omega_{k}^{2}} \max _{\|\mathbf{z}\|=k}|\tilde{r}(\mathbf{z})|^{2} d k
$$

and since $\tilde{r} \in \mathscr{S}\left(\mathbb{R}^{3}\right)$ the limit comparison test with the function $k^{-2}$ permits to positively conclude about the convergence. The expression (A.10) is therefore (proportional to) the limit of Riemann sums of a convergent integral, when the volume of the 3-intervals of the Riemann sums tend to zero. It follows that the infinite volume limit (A.10) exists, and

$$
\lim _{V \rightarrow \infty} \frac{1}{V} \sum_{\mathbf{k} \in \mathcal{R}} \frac{1}{\omega_{k}^{2}}|\tilde{\rho}(\mathbf{k})|^{2}=\frac{1}{(2 \pi)^{3}} \int_{\mathbb{R}^{3}} \frac{1}{\omega_{k}^{2}}|\tilde{r}(\mathbf{k})|^{2} d^{3} k
$$

\section{A.2.2 Potential-mediated expression}

The expression on the right hand side of (A.13) can be recast in a perhaps more intuitive form. Note that, since $r(\mathbf{x}) r(\mathbf{z}) \in C_{c}^{\infty}\left(\mathbb{R}^{6}\right)$, using Fubini's theorem and the definition (A.5) we have

$$
|\tilde{r}(\mathbf{k})|^{2}=\tilde{r}^{*}(\mathbf{k}) \tilde{r}(\mathbf{k})=\iint_{\mathbb{R}^{6}} r(\mathbf{x}) r(\mathbf{z}) \cos [\mathbf{k} \cdot(\mathbf{x}-\mathbf{z})] d^{3} x d^{3} z
$$

SO

$$
\int_{\mathbb{R}^{3}} \frac{1}{\omega_{k}^{2}}|\tilde{r}(\mathbf{k})|^{2} d^{3} k=\int_{\mathbb{R}^{3}} \frac{1}{\omega_{k}^{2}}\left\{\iint_{\mathbb{R}^{6}} r(\mathbf{x}) r(\mathbf{z}) \cos [\mathbf{k} \cdot(\mathbf{x}-\mathbf{z})] d^{3} x d^{3} z\right\} d^{3} k .
$$

It would be attractive to express (A.15) as the integral in $d^{3} x d^{3} z$ of the product of the charge distributions mediated by a potential. As is well-known this is indeed possible, and it results into the appearance of the Yukawa potential mediating the interaction

$$
\int_{\mathbb{R}^{3}} \frac{1}{\omega_{k}^{2}}|\tilde{r}(\mathbf{k})|^{2} d^{3} k=2 \pi^{2} \iint_{\mathbb{R}^{6}} r(\mathbf{x}) r(\mathbf{z}) \frac{e^{-m\|\mathbf{x}-\mathbf{z}\|}}{\|\mathbf{x}-\mathbf{z}\|} d^{3} x d^{3} z
$$

These relations allow us to express the numbers $B$ and $C$ in the forms (2.21) and (2.22). Regarding $C$, consider the expression (2.18) and for each function $\rho_{i}$ introduce the associate auxiliary function $r_{i}$ defined as in section A.1. The relation (A.13) then implies that the infinite volume limit of $C$ takes the form

$$
C \underset{V \rightarrow \infty}{\longrightarrow}-\frac{1}{2} \sum_{i=1}^{2} \frac{g_{i}^{2}}{(2 \pi)^{3}} \int_{\mathbb{R}^{3}} \frac{1}{\omega_{k}^{2}}\left|\tilde{r}_{i}(\mathbf{k})\right|^{2} d^{3} k
$$


and using (A.16) we arrive at

$$
C \underset{V \rightarrow \infty}{\longrightarrow}-\frac{1}{2} \sum_{i=1}^{2} g_{i}^{2} \iint_{\mathbb{R}^{6}} r_{i}(\mathbf{x}) r_{i}(\mathbf{z}) \frac{1}{4 \pi} \frac{e^{-m\|\mathbf{x}-\mathbf{z}\|}}{\|\mathbf{x}-\mathbf{z}\|} d^{3} x d^{3} z
$$

which is $(2.22)$.

Regarding $B$, note that (2.17) can be equivalently written as

$$
B=-\frac{g_{1} g_{2}}{V} \sum_{\mathbf{k} \in \mathcal{R}} \frac{1}{\omega_{k}^{2}} \Re\left[\tilde{\rho}_{1}(\mathbf{k}) \tilde{\rho}_{2}^{*}(\mathbf{k}) e^{-i \mathbf{k} \cdot\left(\mathbf{y}_{1}-\mathbf{y}_{2}\right)}\right],
$$

and that the same arguments of section A.2.1 can be used to infer that its infinite volume limit exists and takes the form

$$
B \underset{V \rightarrow \infty}{\longrightarrow}-\frac{g_{1} g_{2}}{(2 \pi)^{3}} \int_{\mathbb{R}^{3}} \frac{1}{\omega_{k}^{2}} \Re\left[\tilde{r}_{1}(\mathbf{k}) \tilde{r}_{2}^{*}(\mathbf{k}) e^{-i \mathbf{k} \cdot\left(\mathbf{y}_{1}-\mathbf{y}_{2}\right)}\right] d^{3} k
$$

Furthermore, it is straightforward to verify that

$$
\begin{aligned}
& \Re\left[\tilde{r}_{1}(\mathbf{k}) \tilde{r}_{2}^{*}(\mathbf{k}) e^{-i \mathbf{k} \cdot\left(\mathbf{y}_{1}-\mathbf{y}_{2}\right)}\right]= \\
& \quad=\iint_{\mathbb{R}^{6}} r_{1}\left(\mathbf{x}-\mathbf{y}_{1}\right) r_{2}\left(\mathbf{z}-\mathbf{y}_{2}\right) \cos [\mathbf{k} \cdot(\mathbf{x}-\mathbf{z})] d^{3} x d^{3} z,
\end{aligned}
$$

so the integral in (A.20) can be written as

$$
\int_{\mathbb{R}^{3}} \frac{1}{\omega_{k}^{2}}\left\{\iint_{\mathbb{R}^{6}} r_{1}\left(\mathbf{x}-\mathbf{y}_{1}\right) r_{2}\left(\mathbf{z}-\mathbf{y}_{2}\right) \cos [\mathbf{k} \cdot(\mathbf{x}-\mathbf{z})] d^{3} x d^{3} z\right\} d^{3} k .
$$

Analogously to the passage from (A.15) to (A.16), we have

$$
\begin{array}{r}
\int_{\mathbb{R}^{3}} \frac{1}{\omega_{k}^{2}}\left\{\iint_{\mathbb{R}^{6}} r_{1}\left(\mathbf{x}-\mathbf{y}_{1}\right) r_{2}\left(\mathbf{z}-\mathbf{y}_{2}\right) \cos [\mathbf{k} \cdot(\mathbf{x}-\mathbf{z})] d^{3} x d^{3} z\right\} d^{3} k= \\
=2 \pi^{2} \iint_{\mathbb{R}^{6}} r_{1}\left(\mathbf{x}-\mathbf{y}_{1}\right) r_{2}\left(\mathbf{z}-\mathbf{y}_{2}\right) \frac{e^{-m\|\mathbf{x}-\mathbf{z}\|}}{\|\mathbf{x}-\mathbf{z}\|} d^{3} x d^{3} z
\end{array}
$$

so we get

$$
B \underset{V \rightarrow \infty}{\longrightarrow}-g_{1} g_{2} \iint_{\mathbb{R}^{6}} r_{1}\left(\mathbf{x}-\mathbf{y}_{1}\right) r_{2}\left(\mathbf{z}-\mathbf{y}_{2}\right) \frac{1}{4 \pi} \frac{e^{-m\|\mathbf{x}-\mathbf{z}\|}}{\|\mathbf{x}-\mathbf{z}\|} d^{3} x d^{3} z,
$$

which is $(2.21)$. 


\section{References}

[1] Stone, M.H.: Linear transformations in Hilbert space. Operational methods and group theory. Proc. Nat. Acad. Sci. U.S.A. 16, 172 (1930)

[2] Von Neumann, J.: Die Eindeutigkeit der Schrödingerschen Operatoren. Math. Ann. 104, 570 (1931)

[3] Von Neumann, J.: On infinite direct products. Compositio Math. 6, 1 (1939)

[4] Van Hove, L.: Les Difficultés de Divergences pour un Modelle Particulier de Champ Quantifié. Physica 18, 145 (1952)

[5] Friedrichs, K.O.: Mathematical aspects of the quantum theory of fields part IV. Comm. Pure Appl. Math. 5 (4), 349 (1952)

[6] Friedrichs, K.O.: Mathematical aspects of the quantum theory of fields. Interscience Publishers, Inc., New York (1953)

[7] Wightman, A.S., Schweber, S.S.: Configuration Space Methods in Relativistic Quantum Field Theory I. Phys. Rev. 98, 812 (1955)

[8] Lupher, T.: Who Proved Haag's Theorem?. Int. J. Theor. Phys. 44, $1995(2005)$

[9] Earman, J., Fraser, D.: Haag's theorem and its implications for the foundations of Quantum Field Theory. Erkenntnis 64, 305 (2006)

[10] Haag, R.: On quantum field theories. Dan. Mat. Fys. Medd. 29, 1 (1955)

[11] Hall, D., Wightman, A.S.: A theorem on invariant analytic functions with applications to relativistic quantum field theory. Mat. Fys. Medd. Dan. Vid. Selsk. 31, 1 (1957)

[12] Sbisà, F.: On Léon Van Hove's 1952 article on the foundations of Quantum Field Theory. Rev. Bras. Ens. Fis. 42, e20200256 (2020)

[13] Streater, R.F., Wightman, A.S.: PCT, Spin and Statistics, and All That. W.A. Benjamin, Inc., (1964)

[14] Reed, M., Simon, B.: Methods of Modern Mathematical Physics III: Scattering Theory. Academic Press, Inc. (1979)

[15] Haag, R.: Local Quantum Physics, 2nd Edition. Springer-Verlag Berlin Heidelberg (1996)

[16] Thiemann T., Winkler, O.: Gauge Field Theory Coherent States (GCS) : IV. Infinite Tensor Product and Thermodynamical Limit. Class. Quant. Grav. 18, 4997 (2001) 
[17] Loinger, A.: Un semplice modello di due campi interagenti. Il Nuovo Cimento IX, 11, 1080 (1952)

[18] Loinger, A.: Erratum to Un semplice modello di due campi interagenti. Il Nuovo Cimento X, 356 (1953)

[19] Reed M., Simon, B.: Methods of Modern Mathematical Physics II: Fourier Analysis, Self-Adjointness. Academic Press, Inc., (1975) 